\title{
The Patient-Centered Medical Home (PCMH) Framing Typology for Understanding the Structure, Function, and Outcomes of PCMHs
}

\author{
Autumn M. Kieber-Emmons, MD, MPH, and William L. Miller, MD, MA
}

Introduction: Patient-centered medical homes (PCHMs) aspire to transform today's challenged primary care services. However, it is unclear which PCMH characteristics produce specific outcomes of interest for care delivery. This study tested a novel typology of PCMH practice transformation, the PCMH framing typology, and evaluated measurable outcomes by each type.

Methods: Using the Patient-Centered Primary Care Collaborative 2012 to 2013 Annual Review, this secondary analysis of the published PCMH literature extracted data from publications of 59 PCMHs. Each of the 59 sites was categorized as 1 of 4 PCMH types: add-on, renovated, hybrid, or integrated. Six outcome measures (cost reductions, decreased emergency department/hospital utilization, improved quality, improved access, increased preventive services, and improved patient satisfaction) were independently coded for each site. Practices were combined based on type, and mean outcomes scores for each measure were displayed on radar graphs for comparison.

Results: While each type showed a characteristic pattern of success, only the integrated type improved in all 6 outcomes. No type achieved high success in all measures.

Discussion: There seem to be 4 types of PCMH, each of which shows a distinctive outcomes profile. Within the PCMH framing typology, direction is emerging for how best to transform primary care to achieve the greatest success. (J Am Board Fam Med 2017;30:472-479.)

Keywords: Medical Home, Outcome Assessment (Health Care), Patient Satisfaction, Patient-Centered Care, Primary Health Care, Process Assessment (Health Care), Quality Improvement, Quality of Health Care

The 10th anniversary of the patient-centered medical home $(\mathrm{PCMH})$ has arrived. While a host of pilots and demonstrations have been implemented at the practice, network, and state levels over these years, we still do not have full agreement for what a PCMH is. Definitions of the PCMH build on practice characteristics, describing a "place of care integration, family and patient partnership and engagement, operationalization of the primary care core attributes of personal, first contact access,

This article was externally peer reviewed.

Submitted 17 February 2017; revised 18 March 2017; accepted 29 March 2017.

From the Lehigh Valley Health Network, Allentown, PA; and the Department of Family Medicine, University of South Florida Morsani College of Medicine, Tampa.

Funding: none.

Conflict of interest: none declared.

Corresponding author: Autumn M. Kieber-Emmons, MD, MPH, Lehigh Valley Health Netword, One City Center, 707 Hamilton St, Allentown, PA 18101 (E-mail: autumn. kieber-emmons@lvhn.org). comprehensive and coordinated care," but what is emphasized at the practice and health system levels varies considerably throughout the country. ${ }^{1}$ Over the past 10 years, attempts have been made to standardize and evaluate $\mathrm{PCMHs}$, from the $\mathrm{Na}-$ tional Committee for Quality Assurance's metricdriven achievable designations to the Patient-Centered Primary Care Collaborative's (PCPCC) annual reviews of the PCMH evidence. ${ }^{1,2}$ Nonetheless, $\mathrm{PCMH}$ activities still vary with respect to how to study, evaluate, and analyze the transformation to a PCMH and the outcomes these changes have produced.

The central question remains, Which characteristics of a PCMH are particularly linked to producing the outcomes that we are searching for within the triple or quadruple aim? A 2012 tertiary review of the published PCMH literature to date by the second author revealed at that time a possible typology of PCMHs. As reported preliminarily, at 
Table 1. Practice Characteristics Extracted from Published Patient-Centered Medical Home (PCMH) Data and How These Characteristics Overlay to Create the Four Types of PCMHs

\begin{tabular}{|c|c|c|c|c|}
\hline \multirow{2}{*}{$\begin{array}{l}\text { Practice Characteristics Extracted } \\
\text { from Data }\end{array}$} & \multicolumn{4}{|c|}{ Clinic/Practice Typologies } \\
\hline & Add-on (Type 1) & Renovated (Type 2) & Hybrid (Type 3) & Integrated (Type 4) \\
\hline $\begin{array}{l}\text { 1. Added care manager for } \\
\text { specific disease } \\
\text { 2. Care managers for team } \\
\text { 3. Registry capabilities } \\
\text { 4. Scheduling updates } \\
\text { 5. EMR/electronic updates } \\
\text { 6. Care teams } \\
\text { 7. Doctor-task redesign } \\
\text { 8. Payer redesign } \\
\text { 9. Integrated mental health } \\
\text { 10. Community input/community } \\
\text { resource network }\end{array}$ & $\begin{array}{l}\text { 1, } 3 \text {; sometimes may } \\
\text { see } 6 \text { or } 8, \\
\text { specifically } \\
\text { around } 1 \text { disease } \\
\text { type being } \\
\text { managed, (eg, } \\
\text { DM care } \\
\text { management) }\end{array}$ & $\begin{array}{l}\text { Must include some of } \\
2,3,4,5,6,7 \text {, and } \\
8\end{array}$ & $\begin{array}{l}\text { Must include } 1 \text {, plus } \\
\text { some of } 2,3,4,5 \text {, } \\
6,7 \text {, and } 8\end{array}$ & $\begin{array}{l}\text { May include } 1 \text { to } 8 \\
\text { Must include either } \\
9 \text { or } 10\end{array}$ \\
\hline
\end{tabular}

DM, diabetes mellitus; EMR, electronic medical record.

least 4 versions or types of the PCMH may exist, including the "add-on," the "renovated," the "hybrid" stage, and the "integrated" PCMH. ${ }^{3}$ If in fact there are 4 types of PCMHs, analysis of the outcomes profiles attributed to each type would be helpful to health systems and current efforts to transform primary care.

In this article we present our research: a detailed secondary analysis seeking to evaluate the proposed typology hypothesis in question. Through a thorough evaluation of primary data from published articles about PCMHs based on a data set of 59 practices cited in the 2012 to 2013 Patient-Centered Primary Care Collaborative (PCPCC) Annual Review of Evidence, we sought to categorize each of those 59 sites as 1 of the 4 types, then evaluate what outcomes were produced by each type of PCMH. Through the creation of the $\mathrm{PCMH}$ framing typology, as described in this article, we have created a guide for understanding these PCMH characteristics of structure, function, and outcomes. With a clear understanding of what defines each type of $\mathrm{PCMH}$, along with the profile of successful outcomes produced by each type, primary care transformation efforts across the country may be able to focus energies on PCMH characteristics that produce the highest likelihood for success in achieving the triple and quadruple aims.

\section{Methods}

We undertook a detailed secondary analysis of the entirety of the published literature regarding the 59 primary care practices reported in the PCMH Annual Review of Evidence, 2012 to 2013, from the PCPCC. $^{2}$ That report cited 76 published peerreviewed articles or industry press releases. Specific to 2012 to 2013, it was the final year of the Annual Review that included a literature review that culminated in a compilation of the full and comprehensive list of all published new data on PCMHs publically available at that time (for annual reviews after this year, given the rapidly expanding body of published PCMH literature, only additions to the literature in specific categories are highlighted). One author (AMK-E) read all 76 published articles or press releases that were cited in this annual review and extracted data directly from these 76 source publications into an Excel spreadsheet (Microsoft Corp., Redmond, WA) (Tables 1 and 2) before analysis and evaluation. Data extracted from each of the articles included details related to the characteristics and structure of the PCMH itself, as

Table 2. Outcomes Measures Extracted from the Published Patient-Centered Medical Home Data for Each Practice Site

- Cost reductions

- Decreased ED/hospital utilization

- Improved health/quality

- Improved access to care

- Increased preventive services

- Improved patient satisfaction

ED, emergency department. 
well as outcomes reported to date for each site. Outcome measures were categorized into 6 types, in keeping with the same metrics used by the PCPCC in their annual review of the data. These measures were (1) cost reductions; (2) decreased utilization, including both emergency department and hospital admissions; 3) improved health; 4) improved access to care; 5) increased preventive services; and 6) improved patient satisfaction.

From the 76 published peer-reviewed articles or industry press releases, 58 discrete $\mathrm{PCMH}$ clinics or demonstration projects and 1 state employee incentive program were described. Characteristics of each of the 59 sites were extracted from the primary data source, compiling variables aimed at understanding their label within the PCMH framing typology and for the 6 outcomes measures listed above. Only outcomes measures data from published sources were included in this analysis. At that time, data for any of the outcomes measures were not collected from secondary sources beyond these 76 publications. This allowed for the ability to analyze the characteristics and outcomes measures of sites as reported at a static moment in time, and did not introduce bias between sites that may have had updated information publically available compared with other sites that were not yet at that point.

The PCMH framing typology used for defining practices in this study includes the following 4 types of PCMHs: the "add-on," the "renovated," the "hybrid," and the "integrated" (Figure 1). We hypothesized in this PCMH framing typology that a PCMH transformation can occur either by adding a care manager and/or an electronic medical record (EMR) registry to add value to current clinic work processes for particular disease states, or by

Figure 1. Patient-centered medical home framing typology: types 1 to 4 . EMR, electronic medical record.

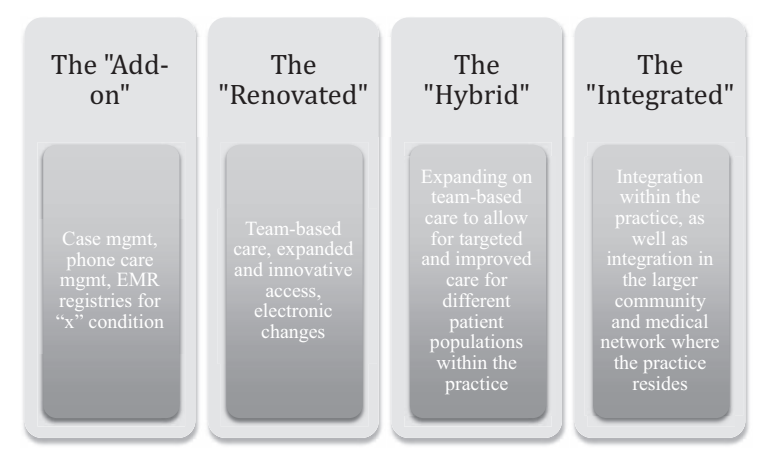

renovating and redesigning the existing work flow of the clinic toward team-based care, including expanded and innovative access points for patients. Some clinics may combine a hybrid of the 2 , using both add-on and renovated aspects to provide more targeted and improved care for different patient populations. Some PCMHs may move further to become integrated within their practice and the local community, through integration with mental health and/or greater inclusion of the larger community and medical neighborhood where the practice resides. These 4 types are descriptive of what has emerged within all the PCMH transformation activity over the past 10 years. The PCMH framing typology is a description of what happened, not necessarily what was intended to occur.

Data collection for the research study began with the development of a list of variables that would allow us to label a clinic or demonstration project along this $\mathrm{PCMH}$ framing typology (see Table 1). These practice characteristics of interest included adding a care manager for specific diseases, care managers for team care, registry capabilities, scheduling updates, EMR/electronic updates, care teams, doctor-task redesigns, payer redesign, integrated mental health, and community input/community resources network. The primary author (AMK-E) then read through each of the primary source documents, extracted all relevant details related to any of these variables, and recorded the data into an Excel database.

Specific combinations of variables are hallmarks of each PCMH type (Table 1). The add-on type requires a site to have added a care manager for specific diseases, often using a registry for specific diseases. This type may sometimes exhibit a care team organized around 1 specific disease and payer redesign focused on that disease, for example, diabetes case management programs. The renovated type requires that a site has substantially redesigned the way care is delivered, with the addition of at least some of the following variables: care managers for team care, registry capabilities, scheduling updates, EMR/electronic updates, care teams, doctortask redesign, and payer redesign. When a practice uses elements of both the add-on and renovated features, the hybrid type of PCMH is realized. Finally, in an integrated PCMH, some or all the first 8 practice characteristics are found, along with an element of integration, through integrated mental health and/or integration with community 
input/community resources network and the medical neighborhood.

Using these specific parameters for each of the PCMH types, both authors coded the 59 sites independently; after analysis of each practice's variables listed in the database, a clinic or demonstration project was labeled as type 1 to 4 within the PCMH framing typology (Figure 1). We then compared and discussed our assigned label for each practice. Any incongruence between our codes was deliberated in detail, and we referenced the primary data source until congruency could be achieved for a label of 1 to 4 .

Outcomes measures, as noted above, were characterized in concordance with the PCPCC's annual review measures of interest and included cost reductions, decreased emergency department and hospital utilization, improved health, improved access to care, increased preventive services, and improved patient satisfaction. ${ }^{2}$ Data were extracted from each of the 76 primary source publications by the first author and placed into each outcomes measure category in the Excel database. To ensure completeness of the secondary literature analysis by the first author in this study, cross-comparisons of the data were analyzed against the outcomes measure categories that were reported as "yes" or "no" by the PCPCC in their annual review document for each of the 59 practice sites. ${ }^{2}$ Discrepancies between our data extracted from the published primary sources and the PCPCC's yes/no categories for each outcome measure were investigated with thorough cross-referencing of primary source publications and confirmed by primary source documentation.

Across the 76 discrete publications, outcomes measures had no standard language or definition in the literature on how to report improvement. A practice may report cost reductions as a return on investment; as a per-member, per-month reduction; as a reduction in total costs; or as a permember, per-year reduction. Most of these figures are unable to be transferred to a common scale for comparison between sites using the published information available publically. To allow for comparison between sites reporting nonstandardized outcomes, we created a weighting system that placed equivalent levels of outcomes achieved at the same numeric score. This coding algorithm assigns a 0,1 , or 2 to each of the 6 outcomes measures; we developed this algorithm to reflect equal weighting across multiple metrics described (Table 3).

The primary author (AMK-E) then analyzed the database for each of the outcomes measures for each practice and gave each site an outcome of 0,1 , or 2 for each of their 6 outcomes measures, depending on how significant the practice's data were in its published studies. The second author then independently reviewed the database and confirmed the appropriate outcome score was awarded for each measure. These scores were based on level of achievement, and not on change, so that practices that were already performing well, with little room for improvement, were so acknowledged. In

Table 3. Outcome Measurements and Coding Algorithm

\begin{tabular}{|c|c|c|}
\hline Cost Reductions & Decreased ED/Hospital Utilization & Improved Quality \\
\hline $\begin{array}{l}\text { - } 0 \text { : Little, no, or negative reductions } \\
\text { in cost }\end{array}$ & - 0 : No or worse utilization reduction & - 0 : No improvement noted \\
\hline $\begin{array}{l}\text { - } 1 \text { : Some improvement noted in cost } \\
\text { reductions }\end{array}$ & $\begin{array}{l}-1: 1-10 \% \text { utilization (ED or admits) } \\
\text { reduction noted }\end{array}$ & $\begin{array}{l}\text { - } 1 \text { : Some improved quality measures } \\
\text { noted }\end{array}$ \\
\hline $\begin{array}{l}\text { - 2: Major improvement in cost } \\
\text { reductions noted ( }>\$ 1 \text { million or an } \\
\text { ROI }>2 \text { or a PMPM or PMPY } \\
\text { reduction }>10 \% \text { or a }>10 \% \\
\text { reduction in total costs) }\end{array}$ & - $2:>10 \%$ utilization reduction noted & $\begin{array}{l}\text { - 2: Health improved dramatically or } \\
\text { all HEDIS measures improved by } \\
\text { at least } 10 \%\end{array}$ \\
\hline Improved Access to Care & Increased Preventive Services & Improved Patient Satisfaction \\
\hline • 0 : No change noted & $\bullet 0$ : No change noted & $\bullet 0$ : No change noted \\
\hline $\begin{array}{l}\text { - 1: The site described a significant } \\
\text { change }\end{array}$ & $\begin{array}{l}\text { - 1: The site described any increase } \\
\text { in preventive services }\end{array}$ & $\begin{array}{l}\text { - 1: The site described a significant } \\
\text { improvement in patient satisfaction }\end{array}$ \\
\hline $\begin{array}{l}\text { - 2: The site described a significant } \\
\text { change and provided meaningful } \\
\text { numbers or data showing } \\
\text { improvement }\end{array}$ & $\begin{array}{l}-2 \text { : The site documented a }>10 \% \\
\text { increase in preventive services }\end{array}$ & $\begin{array}{l}\text { - 2: The site described a documented } \\
>8 \% \text { increase in patient } \\
\text { satisfaction }\end{array}$ \\
\hline
\end{tabular}

ED, emergency department; PMPM, per-member, per-month; PMPY, per-member, per-year; ROI, return on investment. 
addition, to achieve a 2 for improved health, a site had to either show significant improved health among its patient population or have improved all its Healthcare Effectiveness Data and Information Set measures by at least $10 \%$. Only 3 sites achieved this high rating, and we determined that this category should be renamed "improved quality (disease metrics)," given how little emphasis was placed on actual health markers-that is, mortality and morbidity - in the published literature.

After each site was labeled as type 1 to 4 , and after all 6 outcomes measures for each site were given a score of 0,1 , or 2 , we then combined data across each type of PCMH to create radar graphs; these graphs depicted how much and what outcomes were produced by each of the $4 \mathrm{PCMH}$ types. The 59 discrete sites were grouped together based on type, and the mean outcomes scores were calculated for these 4 grouped types. The mean outcomes scores then were used to create 4 radar graphs in order to easily visualize the different metrics on a common scale while allowing for cross-comparison across the PCMH framing typology (Figure 2).

\section{Results}

Of 59 distinct practices or sites discussed in published outcomes studies to date, we found that 9 were the add-on type and 7 were renovated. The large majority of practices or sites $(n=33)$ were a hybrid of both add-on care management and renovated team design. Nine sites showed characteris- tics of an integrated PCMH, in which mental health or social services were truly integrated into the practice itself. Notably, 2 of these 9 integrated sites seemed to transform even further into a $\mathrm{PCMH}$ in which the community itself is an integral force in shaping and implementing its own vision of the medical care system. One site did not fit into the PCMH framing typology system because it was a state-level initiative to incentivize employees to get needed medical services and did not require any PCMH recognition efforts for employees' medical care centers.

Add-on clinics were able to achieve modest success in significantly improving utilization and somewhat improving cost and disease metrics, although they provided little or no improvement for other measures like access or prevention (Figure 2). Renovated clinics, in which a team-based model of care was developed and refined, showed significant improvements in cost, utilization, and access compared with the add-on clinics alone, but they lacked improvement of quality disease metrics or patient satisfaction (Figure 2). Hybrid clinics, a mixture of types 1 and 2, do not seem to be additive in success but mimic the renovated types, albeit with less effect (Figure 2). Integrated clinics show the best balance of outcomes; it was the first of the radar graphs to show improvements in all 6 measures (cost, utilization, access, quality disease metrics, prevention, and patient satisfaction) (Figure 2).

Notably, 2 sites stand out among the type 4 "integrated" sites. In both of these integrated prac-

Figure 2. Radar graphs showing 6 outcomes measures - cost reductions, decreased emergency department/ hospital utilization, improved quality/disease metrics, improved access to care, increased preventive services, and improved patient satisfaction—based on type of patient-centered medical home.

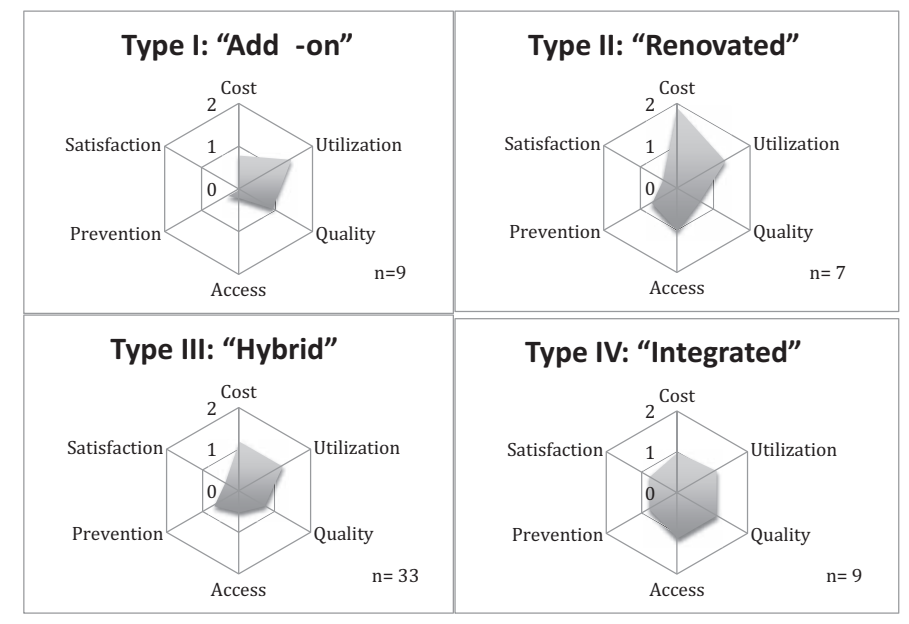


tices, the primary literature revealed a higher level of integration within the community where they reside. In essence these $2 \mathrm{PCMHs}$ transformed into something more, paramount to a community-centered medical home. One site, SouthCentral Alaska Nuka Foundation, evolved from the ashes of a crumbling medical system into a health infrastructure where the local patients, called "customer owners," define their needs, goals, and values and have transformed their health care from the inside out. Having the patient community be the leadership for the health system directs the focus on patient preferences for care decisions, and local needs influence what clinicians emphasize for care. ${ }^{4}$ A second example of how a PCMH can transform to a more community-minded integration is found in Texas, through a nonprofit health system called WellMed. This primary-care based accountable care organization, caring for $\geq 87,000$ Medicare Advantage members, effectively decreased mortality by $50 \%$ compared with controls, through a sophisticated design of outpatient ambulatory wrap-around services and connections in the community, for instance, through provision of medical transport services, mobile EMR bracelets, and elderly health-improving community agencies. ${ }^{5}$

\section{Discussion}

How do we know the PCMH will actually be successful? This question has yet to be definitively answered in today's era of rapidly changing health care reform. While the PCMH concept aspires to fulfill the triple and quadruple aims and transform today's challenged primary care services, there are still unclear expectations of how a PCMH demonstrates success, on what scales, and with which outcomes. The PCMH framing typology aims to provide an understanding of how PCMHs are currently developing and of what aspects and qualities of a PCMH produce the largest positive outcomes in cost, utilization, access, quality, prevention, and satisfaction. In addition, it is an important objective of primary care to be always striving to optimize and improve a multiplicity of goals. As we search for the best paradigm for care transformation, we suggest not only that primary care's efforts should ideally produce 1 or 2 positive outcomes, but alsoand more importantly-that the primary care roadmap that achieves the most success among the diverse group of outcomes be our target.
Our results of the PCMH framing typology describe the 4 types of PCMHs, their characteristics, and the outcomes achievable with each type (Figure 2). As one might anticipate, the add-on PCMH care manager function (type 1) is just that-an à la carte addition to a currently functioning primary care clinic aimed at improving care for a small segment of that clinic's patient population. A care manager for superutilizers, patients with diabetes, or recently hospitalized patients will likely improve outcomes of cost or utilization for that group of patients, but will not significantly affect the other outcomes. Using a team-based care approach, with EMR and access changes, the renovated PCMH (type 2) shows a significant improvement in cost and some improvement in utilization and access. Our data support the proponents of primary care redesign, showing that teamwork does pay, but maybe not as much as may have been anticipated. Interestingly, the hybrid PCMH (type 3) was not simply an additive function of types 1 plus 2 ; rather, positive outcomes decreased when case management and team redesign were merged. One explanation for this decreased effectiveness may be the concept of change fatigue and burnout, described in the published literature, whereby extending the focus to more clinic-based tasks, creating longer lists of practice improvements, and redefining employee roles more significantly delays or lessens the impact on outcomes. ${ }^{6}$

Integrated PCMHs (type 4) seem to be most successful in terms of a balanced and comprehensive improvement in all 6 outcomes measures (Figure 2). Evaluation of these clinics suggest that by becoming a PCMH that was integrated with mental health or other community agencies, these practice sites were able to achieve the best of all worlds: decreasing costs and utilization while improving access, disease metrics, and preventive services, and attaining patient satisfaction-although none of the 6 outcomes get much beyond a score of 1 . When these data are combined with the known significant cost of implementing PCMH changes, the challenges facing sustainable PCMH transformation become daunting. ${ }^{7}$

One site listed in the annual review did not fit into the PCMH framing typology. This site was a state-level payment reform initiative that encouraged patients to get needed preventive and healthy living testing, regardless of what type of clinic or PCMH they actually belonged to. Interestingly, 
this site did show notable positive outcomes measures, regardless of PCMH status, achieving a score of 2 in both cost and utilization reductions, and a score of 1 in improved access and improved preventive services. It seems that by directly motivating patient employees themselves with payment reform, impressive cost and utilization reductions as well as improved access and preventive services may also be achievable, regardless of whether clinics are trying to become a PCMH. Such solo payment reforms have interesting implications for current policymakers, as we continue to search and refine what makes for the "best primary care" infrastructure and delivery system nationally. This particular model suggests an additional method to consider as a supplement to the PCMH for achieving the triple and quadruple aims, and illuminates our need for continued discussion on payment reform and how it may be a crucial step in primary care transformation.

Furthermore, we emphasize that many practices remarked on the incredible patience that must be exhibited to travel the road to becoming a PCMH. Practice transformation takes time and effort. It may even be detrimental or unsuccessful at different times in its evolution. As an additional analysis of our model, we looked at data outcomes published 1 year after this study data set by reading the source publications noted in the January 2015 PCPCC's Annual Review of Evidence, 2013 to $2014 .^{8}$ Interestingly, many of the insurance and large health system improvements highlighted in this edition of the annual review point out that cost and utilizations savings did indeed only become realized after $\geq 3$ years of practice transformation work. $^{9-11}$

Much research and energy are currently being invested nationally on primary care transformation. "The 10 Building Blocks of High-Performing Primary Care" described by Bodenheimer et $\mathrm{al}^{12}$ is 1 such research model that provides guidance for practices trying to achieve the triple and quadruple aims. In comparing the PCMH framing typology against the building block model, it becomes apparent that these models, when evaluated in tandem, crystalize an understanding of the steps and processes necessary for transformation. For instance, in our add-on stage, partial improvements are made in building blocks 1 (engaged leadership), 2 (data-driven improvement), 3 (empanelment), and 6 (population management). The hybrid stage includes total improvement in blocks 1 (engaged leadership) and 2 (data-driven improvement) and partial improvement in blocks 3 (empanelment), 4 (team-based care), 6 (population management), and 8 (prompt access to care). The integrated practice, type 4 , shows the most building blocks achieved, with total improvement of blocks 1 (engaged leadership), 2 (data-driven improvement), 3 (empanelment), and 4 (team-based care) and partial improvement in blocks 5 (patient-team partnership), 6 (population management), 8 (prompt access to care), and 9 (comprehensiveness and care coordination). Our study and the PCMH framing typology describe what has actually emerged as practices have worked to become PCMHs, whereas the building block model describes what has worked best. Notice that access and population management are often implemented before the initial building blocks are solidly in place. This might partly explain the less-than-inspiring results seen in our study.

\section{Limitations}

Our study has a few limitations, specifically with regard to characterization of practices into both types and outcomes reported. Given that this study looked only at the primary literature reported in the 2012 to 2013 PCPCC annual review, a practice may have had outcomes measures available but not published at that time. Another major source of variation, which we attempted to control for with the radar graphs and by plotting all measures on a 0 -to-2 scale, is the lack of a common vernacular in terms of how practices go about reporting data. Thus, a return on investment of $4 \times$ may or may not be the same order of magnitude improvement as $\$ 1$ million in annual savings; for the purposes of this study, however, we developed somewhat arbitrary cut points for categories 1 and 2. It is hoped that, as we continue to develop and refine outcomes measures nationally, a common set of metrics will be agreed on to allow simplified comparisons between practices. ${ }^{13} \mathrm{We}$ also need systematic measures of better health and practice joy to measure where PCMHs are achieving these goals.

\section{Conclusions}

After 10 years, what next for the PCMH? We believe that enough is now known for both policymakers and primary care leaders to pause and re- 
evaluate this question. Much progress has been made, and extensions of the current PCMH should continue to evolve and be evaluated. We also recommend more active pursuit of alternative models. Through the PCMH framing typology, 4 types of PCMHs are emerging, and each results in a different outcomes profile. None of them are yet achieving outstanding success, although the integrated PCMH seems to be having a positive effect on all the assessed goals for primary care. We have not yet achieved transformation, but hopeful signs and directions are emerging.

To see this article online, please go to: bttp://jabfm.org/content/ 30/4/472.full.

\section{References}

1. Miller WL. Patient-centered medical home (PCMH) recognition: a time for promoting innovation, not measuring standards. J Am Board Fam Med 2014;27: 309-11.

2. The medical home's impact on cost \& quality: an annual updated of the evidence 2012-2013. PatientCentered Primary Care Collaborative, January 2014. Available from: https://www.pcpcc.org/resource/ medical-homes-impact-cost-quality. Accessed June 13, 2017.

3. Sakallaris B, Miller W, Saper R, Kreitzer M, Jonas $W$. Meeting the challenge of a more person-centered future for US healthcare. Glob Adv Health Med 2016;5:51-60.

4. Driscoll DL, Hiratsuka V, Johnson JM, et al. Process and outcomes of patient-centered med care with Alaska Native people at Southcentral Foundation. Ann Fam Med 2013;11(Suppl 1):S41-9.

5. Phillips RL, Bronnikov S, Petterson S, et al. Case study of a primary care-based accountable care system approach to medical home transformation. J Ambul Care Manage 2011;34:67-77.

6. Nutting P, Miller W, Crabtree B, Jaen C, Stewart E, Stange K. Initial lessons from the first national dem- onstration project practice transformation to a patient-centered medical home. Ann Fam Med 2009; 7:254-60.

7. Magill M, Ehrenberger D, Scammon DL, et al. The cost of sustaining a patient-centered medical home: experience from 2 states. Ann Fam Med 2015;13: 429-35.

8. Nielson M, Gibson L, Buelt L, Grundy P, Grumbach K. The patient-centered medical home's impact on cost and quality: annual review of evidence, 2013-2014. Patient-Centered Primary Care Collaborative, January 2015. Available from: https:// www.pcpcc.org/resource/patient-centered-medicalhomes-impact-cost-and-quality-annual-reviewevidence-2013-2014. Accessed June 13, 2017.

9. Phillips RL, Han M, Petterson SM, Makaroff LA, Liaw WR. Cost, utilization, and quality of care: an evaluation of Illinois' Medicaid primary care case management program. Ann Fam Med 2014;12: 408-17.

10. UnitedHealth Group. Advancing primary care delivery: practical, proven, and scalable solutions. Available from: www.unitedhealthgroup.com/ /media/UHG/ PDF/2014/UNH-Primary-Care-Report-AdvancingPrimary-Care-Delivery.ashx. Accessed June 20, 2017.

11. Harmen JS, Hall AG, Lemak CH, Duncan PR. Do provider service networks result in lower expenditures compared with HMOs or primary care case management in Florida's Medicaid program? Health Serv Res 2014;49:858-77.

12. Bodenheimer T, Ghorob A, Willard-Grace R, Grumbach K. The 10 building blocks of high-performing primary care. Ann Fam Med 2014;12:166-71.

13. Peikes D, Taylor E, Genevro J, Meyers D. A guide to real-world evaluations of primary care interventions: some practical advice. October 2014 AHRQ pub no. 14-0069-EF. Available from: https://www. mathematica-mpr.com/our-publications-and-findings/ publications/a-guide-to-realworld-evaluations-ofprimary-care-interventions-some-practical-advice. Accessed June 20, 2017. 